\title{
Potassium Phosphate, Dibasic
}

National Cancer Institute

\section{Source}

National Cancer Institute. Potassium Phosphate, Dibasic. NCI Thesaurus. Code C66452.

The dipotassium form of phosphoric acid, that can be used as an electrolyte replenisher and with radio-protective activity. Upon oral administration, potassium phosphate is able to block the uptake of the radioactive isotope phosphorus $\mathrm{P} 32$ (P-32). 